CERN-TH.7226/94

\title{
SUPERGRAVITY BEFORE AND AFTER 1976
}

\author{
D.V. Volkov*) \\ K.F.T.I., Kharkov, Ukraine \\ and \\ Theoretical Physics Division, CERN \\ CH - 1211 Geneva 23
}

\begin{abstract}
This paper is part of the lecture given at the TH Division of CERN and devoted to the CXXV anniversary of the birthday of Elie Cartan (1869-1951). It is shown how the methods of differential geometry, due to E. Cartan, were applied to the construction of the supersymmetry transformation law and to the actions for Goldstone fermions and supergravity.

*) Permanent address: Kharkov Institute of Physics and Technology, Kharkov 310108, Ukraine. e-mail address: dvolkov@ kfti.kharkov.ua.
\end{abstract}

CERN-TH.7226/94

April 1994 
In the review articles and monographs on supergravity, its discovery is usually dated as 1976. However, a number of papers on supergravity appeared before 1976, beginning from 1972 [1]-[5]. As has now been confirmed, those papers are directly related to the recognized version of supergravity [6],[7] and in some sense were the starting points for the later ones. In this paper, I will try to fill in the historical gap (1972-1976).

Supergravity is the gauged version of the global supersymmetry. Therefore I will begin by exposing briefly those elements of supersymmetry which will be essential for the following.

Supersymmetry has been independently discovered by three groups of authors: Yu. Gol'fand and E. Lichtman [8]; D. Volkov and V. Akulov [1]; J. Wess and B. Zumino [9]. The motivations and starting points used by the three groups of authors were quite different.

In Ref. [8] the motivation was to introduce a parity violation into the quantum field theory. The starting point of the papers [1],[2] was the question whether Goldstone particles with spin one-half might exist. The authors of Ref. [9] made the generalization of the supergroup which first appeared in the Neveu-Ramond-Schwarz dual model [10],[11] to the four-dimensional world.

The approach of the papers [1],[2] was the most appropriate for gauging the super-Poincaré group which was done a little later in the papers of D. Volkov and V. Soroka [3],[4] (1973-1974), where the super-Higgs effect in supergravity was elaborated.

The connection of the papers [1],[2] and [3],[4] is very natural, as in the gauge field theories the transformation law for the gauge fields is determined by the same group structure which gives a description of the Goldstone fields. So I will consider, as an introduction, those features of the supersymmetry theory that are essential for its gauging.

A detailed exposition of the route along which the generalization of the Poincare group to the super-Poincaré group was made is contained in Ref. [2]. As this paper is not well known, I will briefly recall its most essential points.

As has been mentioned above, the starting point was the question whether Goldstone particles with spin one-half might exist. At the end of the Sixties, the method of the phenomenological Lagrangians for the description of Goldstone particles, so that it reproduced the results of PCAC (partially conserved axial-vector currents) and the current algebra, had been invented by S. Weinberg and J. Schwinger. At the time of the XIVth Conference on high-energy physics (Vienna, 1968), the problem of the current algebra and of the phenomenological Lagrangians had been intensively discussed (see Weinberg's rapporteur talk [12]). There were two papers presented in the current algebra section of the conference in which the generalization of the method of phenomenological Lagrangians to an arbitrary internal symmetry group had been elaborated. One paper was presented by B. Zumino [13] (co-authors C. Callan, S. Coleman and J. Wess), and another one by myself [14]. The main results of the papers were practically identical. The difference was that in Ref. [14] ${ }^{1}$ the works of E. Cartan on symmetric spaces and his method of the exterior differential forms was intensively used.

\footnotetext{
${ }^{1}$ In [15], which contains [14], the methods of E. Cartan was also used for the construction of phenomenological Lagrangians for the spontaneously broken symmetry groups, containing the Poincaré group as a subgroup. The Lagrangian for the Goldstone fermions (9) is an example of such a construction.
} 
In both papers, as well as in E. Cartan's works ${ }^{2}$, the decomposition of a group $G$ on the factors

$$
G=K H
$$

was used, with the parameters of the coset $K$ forming a homogeneous space, and $H$ being the holonomy group of the space.

In the method of the phenomenological Lagrangians, the co-ordinates of the coset $K$ correspond to the Goldstone fields. Therefore the quantum numbers of Goldstone fields coincide with the quantum numbers of the generators of the coset $K$. This fact gives the answer to the question about the possibility of the existence of Goldstone particles with spin one-half.

To ensure such a possibility, the Poincaré group should be generalized in such a way that the generalization contains the generators with spin one-half and with commutation relations corresponding to the Fermi statistics. From a technical point of view the problem was what representation of the Poincaré group is the most appropriate for such a generalization.

The solution of this technical problem was that the following representation of the Poincaré group

$$
G_{\text {Poincare }}=\left(\begin{array}{cc}
L & i X L^{+-1} \\
0 & L^{+-1}
\end{array}\right)=\left(\begin{array}{cc}
1 & i X \\
0 & L
\end{array}\right)\left(\begin{array}{cc}
L & 0 \\
0 & L^{+-1}
\end{array}\right)
$$

where $L, L^{+-1}$ and $X$ are the $2 \times 2$ matrices $L=L_{\alpha}{ }^{\beta} ; L^{+-1}=L_{\dot{\beta}}^{\dot{\alpha}} ; X=X_{\alpha \dot{\beta}}$, had all required properties.

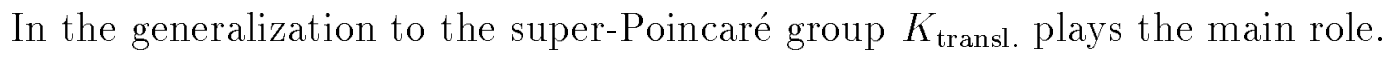

Let us write it as consisting of four blocks:

$$
K=\left(\begin{array}{c|c}
1 & \mathrm{iX} \\
\hline 0 & 1
\end{array}\right)
$$

Separating the blocks as follows

$$
K^{\prime}=\left(\begin{array}{c|c|c}
1 & - & \mathrm{iX} \\
\hline 0 & 1 & - \\
\hline 0 & 0 & 1
\end{array}\right)
$$

one can insert into the newly formed hatched blocks Grassmann spinors $\theta_{\alpha}$ and $\bar{\theta}_{\dot{\alpha}}$ so that $K^{\prime}$ becomes

$$
K^{\prime}=\left(\begin{array}{c|c|c}
1 & \theta & \mathrm{iX} \\
\hline 0 & 1 & \theta \\
\hline 0 & 0 & 1
\end{array}\right)
$$

The matrix $K^{\prime}$ forms a group, but only under the condition that $X^{\prime}$ is complex. To satisfy the reality condition for $X^{\prime}$ with the reality condition for $X$ in (2) and simultaneously conserving

\footnotetext{
${ }^{2}$ And also in the many papers that followed.
} 
the group properties of (5), the following representation of $X^{\prime}$, as a sum of real and imaginary parts, is appropriate

$$
i X^{\prime}=i X+\frac{1}{2} \theta \bar{\theta}
$$

The resulting expression for the super-Poincaré group is the following

$$
G_{\mathrm{SUSY}}=\left(\begin{array}{ccc}
1 & \theta & i X+\frac{1}{2} \theta \bar{\theta} \\
0 & 1 & \bar{\theta} \\
0 & 0 & 1
\end{array}\right)\left(\begin{array}{ccc}
L & 0 & 0 \\
0 & 1 & 0 \\
0 & 0 & L^{+-1}
\end{array}\right)
$$

From (15) one gets the transformation law for the superspace coordinates

$$
\begin{aligned}
X^{\prime} & =X+i \epsilon \bar{\theta} \\
\theta^{\prime} & =\theta+\epsilon ; \quad \bar{\theta}^{\prime}=\bar{\theta}+\bar{\epsilon}
\end{aligned}
$$

as well as the following expressions for the left-invariant vielbein one-differential forms on the superspace $(X, \theta)$

$$
\begin{aligned}
e^{a} & =d X^{a}+i \bar{\theta} \gamma^{a} d 0 \\
e^{\alpha} & =d \theta
\end{aligned}
$$

The latter are received as components of $K^{-1} d K$ corresponding to the generators of $K$ - now being the supertranslation subgroup of $(6)$.

The action for the Goldstone fermions is an integrated four-form pulled back onto the fourdimensional Minkowski space (the world space) and

$$
L_{G F}=\frac{1}{24} \epsilon_{a b c d} e^{a} e^{b} e^{c} e^{d}
$$

The expressions (7)-(9) were written in [1] without the detailed deduction which was later reproduced in $[2]$.

Now, as a first step in discussing the problem of supersymmetry gauging, I cite the final sentence of [1]:

“...the gravitational interaction may be included by means of introducing the gauge fields for the Poincaré group. Note that if the gauge field for the transformation (3) [formula (7) of the present text] is also introduced, then as a result of the Higgs effect the massive gauge field with spin three-halves appears and the considered Goldstone particle with spin one-half disappears."

Now let us go to the procedure of gauging the supersymmetry. 
The gauge fields for the local supersymmetry group can be introduced in the standard way

$$
A_{\mathrm{SUSY}}(d)=\left(\begin{array}{ccc}
\omega_{\alpha}{ }^{\beta}(d) & \psi_{\alpha}(d) & e_{\alpha \dot{\beta}}(d) \\
& 1 & \bar{\psi}_{\dot{\beta}}(d) \\
& & \omega_{\dot{\beta}}^{\alpha}(d)
\end{array}\right)
$$

with the standard transformation law

$$
A^{\prime}(d)=G^{-1} A(d) G+G^{-1} d G
$$

where $A(d)$ is the $g_{\mathrm{SuSY}}$-algebra valued one-differential forms.

Expression (11) may be interpreted in two ways: the first one as the transformation law for $A(d)$, and the second one in which $G$ or some of its coset $K$ is interpreted as the space of Goldstone fields.

In the latter case, if the Goldstone fields transform as $G_{L}^{\prime}=L G$ ( $L$ is the left multiplication on the group $G$ ) then Eq. (11) is invariant.

Analogously, if the coset $K$ contains the Goldstone fields, then $K_{L}^{\prime}=L K H^{-1}(K, L)$

$$
A^{\prime}(d)=H\left(K^{-1} A K+K^{-1} d K\right) H^{-1}+H d H^{\prime}
$$

so that the projections of $K^{-1} A K+K^{-1} d K$ on the generators of $K$ are the covariants of the subgroup $H$.

The second way of interpreting (11) is appropriate for considering the spontaneously broken supergravity; the first one is useful if pure (not broken) supergravity is considered.

In our case, the forms $e_{\alpha \dot{\beta}}(d) \psi_{\alpha}(d)$ and $\bar{\psi}_{\dot{\alpha}}(d)$, as well as the curvature tensor $R_{\beta}^{\alpha}\left(d, d^{\prime}\right)$; $R_{\dot{\beta}}^{\dot{\alpha}}\left(d, d_{z}^{\prime}\right)$ for the Lorentz connection $\omega(d)$, are the covariants of the Lorentz subgroup $L$.

The covariant forms (10) in the presence of the Goldstone fields $X^{a}, \theta^{\alpha}$ and $\theta^{\dot{\alpha}}$ may be written as

$$
\begin{aligned}
& \tilde{e}(d)=e(d)+\mathcal{D} X+i[(2 \psi(d)+\mathcal{D} \theta) \bar{\theta} \theta(2 \bar{\psi}(d)+\mathcal{D} \bar{\theta})] \\
& \tilde{\psi}(d)=\psi(d)+\mathcal{D} \theta \\
& \tilde{\omega}(d)=\omega(d) \\
& \tilde{R}\left(d, d^{\prime}\right)=R\left(d, d^{\prime}\right)=d \omega\left(d^{\prime}\right)-d^{\prime}\left[\omega(d), \omega\left(d^{\prime}\right)\right]
\end{aligned}
$$

Note that in the case of infinitesimal transformation only terms linear in $\theta$ are present in (13).

One can now construct the following invariant four-differential forms contracting the indices of the one-differential forms [3],[4]

$$
\begin{aligned}
W_{1} & =R\left(d_{1}, d_{2}\right) e\left(d_{3}\right) e\left(d_{4}\right) \\
W_{2} & =\mathcal{D} \bar{\psi}\left(d_{1}, d_{2}\right) e\left(d_{3}\right) \psi\left(d_{4}\right)
\end{aligned}
$$




$$
\begin{gathered}
W_{3}=e\left(d_{1}\right) e\left(d_{2}\right) e\left(d_{3}\right) e\left(d_{4}\right) \\
W_{4}=\bar{\psi}\left(d_{1}\right) e\left(d_{2}\right) e\left(d_{3}\right) \psi\left(d_{4}\right)
\end{gathered}
$$

using either the spinor notation $\left(\alpha, \dot{\alpha}\right.$ - indices) or the more usual vector notation for $R\left(d_{1}, d_{2}\right)$ and $e(d)$, so that $W_{1}, W_{2}, W_{3}$ and $W_{4}$ represent correspondingly the Einstein action, the RaritaSchwinger kinetic term, the cosmological term and the mass term for the Rarita-Schwinger field.

The resulting action is the sum

$$
W=\underbrace{a_{1} W_{1}+a_{2} W_{2}}_{\text {the pure SUGRA }}+\underbrace{a_{3} W_{3}+a_{4} W_{4}}_{\begin{array}{c}
\text { the terms due to the } \\
\text { spontabreakdown of } \\
\text { the super - Poincare group }
\end{array}}
$$

The fact that the sum $a_{1} W_{1}+a_{2} W_{2}$ is the pure unbroken supergravity follows from counting the degrees of freedom of the Rarita-Schwinger field with the action $W_{2}$ in a gravitational background. It is easy to show that if the gravitational background satisfies the equations of motion for the Einstein action $W_{1}$, then the Rarita-Schwinger field has two degrees of freedom. So the Goldstone fields do not contribute on the mass shell of the gravitational field.

The formulas (14) and (15) and transformation law (13) are the main results of the papers $[3],[4]$.

Now let us turn to the works on supergravity that appeared in 1976 . The first of these works [6] used the second-order formalism for the Einstein action with a rather complicated transformation law for the supergravity gauge fields. A more simplified form of the action and transformation law has been proposed in the paper of Deser and Zumino [7]. These authors have written in their introduction:

"The key to our results lies in the use of the first-order formalism for gravitation, in which vierbeins and connection coefficients are treated independently. Minimal coupling in this sense implies the existence of torsion, or of non-minimal contact interactions in second-order language. The first-order formulation with torsion is closely related to the description of supergravity in superspace $[5]^{3}$."

The transformation laws for the gauge fields $e(d)$ and $\psi(d)$ which were used in the paper of Deser and Zumino [7] coincided with (22a,b) but were different from (22c) for the Lorentz connection form $\omega(d)$. The further development of the supergravity theory has shown that the explicit forms of the $\delta \omega$ variation does not matter.

As a result of further investigations, the first-order formalism and the gauged supergroup approach to the supergravity based on the transformations (13) is now accepted to be the

\footnotetext{
${ }^{3}$ The reference note is given according to the list of references of the present paper. In the paper referred to, E. Cartan's methods of differential geometry are firstly generalized to the graded superspaces. It is also shown that "the flat superspace" has torsion, and that the holonomy group for the curvature in the superspace formulation of supergravity should be the Lorentz group.
} 
simplest way to supergravity ${ }^{4}$. The important steps along this line of reasoning were the rheonomy theory of supergravity as well as the above-mentioned explicit proofs of the invariance of the supergravity action by using the transformation law (13), and showing that the condition $\delta \omega(d)=0(13 \mathrm{c})$ considerably simplifies the proof. The advantages of the first-order formalism were proved to be useful in many aspects of the theory.

The period of intensive development of supergravity since 1976 is well described in the review article [16] and others which followed. In the text of the review [16] there is no reference to the papers [1]-[5]. So the present paper may be considered as an addendum to [16] with reference to the papers which were published before 1976 and were essentially based on the first-order formalism, developed, in its main features, by E. Cartan.

\section{Acknowledgements}

I am grateful to the Theoretical Physics Division for kind hospitality during my stay at CERN. This work was partially supported by the International Science Foundation and Ukrainian State Committee in Science and Technologies, Grant Nr 2/100.

\footnotetext{
${ }^{4}$ See, for example, [16].
} 


\section{References}

[1] D. Volkov and V. Akulov, JETP Lett. 16 (1972) 438; Phys.Lett. 46B (1973) 109.

[2] V. Akulov and D. Volkov, Theor.Math.Phys. 18 (1974) 28.

[3] D. Volkov and V. Soroka, JETP Lett. 18 (1973) 312.

[4] D. Volkov and V. Soroka, Theor.Math.Phys. 20 (1974) 829.

[5] V. Akulov, D. Volkov and V. Soroka, JETP Lett. 22 (1975) 187.

[6] S. Ferrara, D. Freedman and P. Van Nieuwenhuizen, Phys.Rev. D13 (1976) 3214.

[7] S. Deser and B. Zumino, Phys.Lett. 62B (1976) 335.

[8] Yu. Gol'fand and E. Lichtman, JETP Lett. 13 (1971) 323.

[9] J. Wess and B. Zumino, Nucl.Phys. B70 (1974) 39.

[10] A. Neveu and J. Schwarz, Nucl.Phys. B31 (1971) 86;

R. Ramond, Phys.Rev. D3 (1971) 2415.

[11] J.-L. Gervais and B. Sakita, Nucl.Phys. B34 (1971) 633.

[12] S. Weinberg, Proceedings of the XIV International Conference on High Energy Physics, Vienna, 1968.

[13] S. Coleman, J. Wess and B. Zumino, Phys.Rev. 177 (1969) 2239;

C. Callan et al., Phys.Rev. 177 (1969) 2247.

[14] D. Volkov, Kiev Preprint ITF-69-75 (1969).

[15] D. Volkov, Soviet J.Part.Nucl. 4 (1973) 1.

[16] P. Van Nieuwenhuizen, Physics Reports 68 (1981) 191. 